\title{
Candidate gene prioritization by network analysis of differential expression using machine learning approaches
}

\author{
Daniela Nitsch ${ }^{1 *}$, Joana P Gonçalves ${ }^{1,2,3}$, Fabian Ojeda ${ }^{1}$, Bart de Moor ${ }^{1}$, Yves Moreau ${ }^{1}$
}

\begin{abstract}
Background: Discovering novel disease genes is still challenging for diseases for which no prior knowledge - such as known disease genes or disease-related pathways - is available. Performing genetic studies frequently results in large lists of candidate genes of which only few can be followed up for further investigation. We have recently developed a computational method for constitutional genetic disorders that identifies the most promising candidate genes by replacing prior knowledge by experimental data of differential gene expression between affected and healthy individuals.

To improve the performance of our prioritization strategy, we have extended our previous work by applying different machine learning approaches that identify promising candidate genes by determining whether a gene is surrounded by highly differentially expressed genes in a functional association or protein-protein interaction network.

Results: We have proposed three strategies scoring disease candidate genes relying on network-based machine learning approaches, such as kernel ridge regression, heat kernel, and Arnoldi kernel approximation. For comparison purposes, a local measure based on the expression of the direct neighbors is also computed. We have benchmarked these strategies on 40 publicly available knockout experiments in mice, and performance was assessed against results obtained using a standard procedure in genetics that ranks candidate genes based solely on their differential expression levels (Simple Expression Ranking). Our results showed that our four strategies could outperform this standard procedure and that the best results were obtained using the Heat Kernel Diffusion Ranking leading to an average ranking position of 8 out of 100 genes, an AUC value of 92.3\% and an error reduction of $52.8 \%$ relative to the standard procedure approach which ranked the knockout gene on average at position 17 with an AUC value of $83.7 \%$.
\end{abstract}

Conclusion: In this study we could identify promising candidate genes using network based machine learning approaches even if no knowledge is available about the disease or phenotype.

\section{Background}

Discovering novel disease genes is still challenging for constitutional genetic diseases (a disease involving the entire body or having a widespread array of symptoms) for which no prior knowledge - such as known disease genes or disease-related pathways - is available. Performing genetic studies frequently result in large lists of candidate genes of which only few can be followed up for further investigation. Gene prioritization establishes the

\footnotetext{
* Correspondence: Daniela.Nitsch@esat.kuleuven.be

'Department of Electrical Engineering (ESAT-SCD) Katholieke Universiteit Leuven, 3001 Leuven, Belgium

Full list of author information is available at the end of the article
}

ranking of candidate genes based on their relevance with respect to a biological process of interest, from which the most promising genes can be selected for further analysis.

Several computational methods for prioritizing candidate genes have been proposed, such as ENDEAVOUR [1], GeneWanderer [2], or Prioritizer [3] that rank candidate genes based on associations between known disease genes and candidate genes using different data sources and methodology. Lage et al. (2007) developed a phenome-interactome network that integrates phenotypic literature information from OMIM with a cross species protein-protein interaction (PPI) network [4]. Chen
C Biomed Central

C 2010 Nitsch et al; licensee BioMed Central Ltd. This is an Open Access article distributed under the terms of the Creative Commons Attribution License (http://creativecommons.org/licenses/by/2.0), which permits unrestricted use, distribution, and reproduction in any medium, provided the original work is properly cited. 
et al. (2009) applied link based strategies widely used in social and web network analyses to prioritize disease candidate genes based on PPI networks [5]. Subramanian et al. (2005) developed a computational method, Gene set enrichment analysis (GSEA), that determines whether an a priori defined set of genes shows statistically significant concordant differences between two biological states (e.g. phenotypes), i.e. whether the genes are differentially expressed or not [6]. Similarly, Liu et al. (2007) developed a network-based approach, Gene Network Enrichment Analysis (GNEA) for the identification of transcriptionally altered biological processes between disease and normal states [7]. It analyzes gene expression microarray and protein-protein interaction data to identify the affected regions in a protein-protein interaction network. Beside these methods, others can be found in the literature that prioritize candidate genes for human diseases. Among these, several were implemented as web-based applications that can be freely accessed. In a previous study we have reviewed distinct web-based and freely accessible gene prioritization tools for human diseases [8].

The aforementioned methods usually rank candidate genes by matching their information across multiple data sources against a profile derived from a set of genes, keywords, pathways, or biological processes already known to be associated with the phenotype. However, often only little is known about the molecular basis of the phenotype, such as no known disease genes and limited knowledge about the biological cascades involved, and only few or no keywords are known which can be used either to retrieve genes or to perform text mining. To overcome this limitation, we proposed in a previous study a computational method to identify the most promising candidates within a region for which limited or no prior knowledge is available regarding a phenotype of interest by using experimental data on differential gene expression between affected and healthy individuals [9]. Using a network-based approach, we assessed the relevance of a candidate gene by considering the level of differential expression in its neighborhood under the assumption that strong candidates tend to be surrounded by differentially expressed neighbors. For several genetic diseases, however, there is no guarantee that the expression levels of the disease gene itself is affected, rather, genes "downstream" of the disease gene are those whose expression will be affected. Therefore, we consider the differential expression data at the network levels instead of (isolated) gene levels. Mapping expression patterns on a network, we then expect to observe a disrupted expression module around the disease gene, while other candidate genes (not causally related to the phenotype) should not be part of such a disrupted expression module. This approach is less biased than assessing relevant candidate genes by performing text mining, finding associations between known disease genes and candidate genes, or detecting disease relevant pathways. We define a notion of a soft neighborhood where each gene is given a contributing weight, which decreases with the distance from the candidate gene in the network. To account for multiple paths between genes, we defined a notion of distance using the Laplacian exponential diffusion kernel. Finally, we scored candidates by aggregating the differential expression of their neighbors weighted as a function of distance.

To further improve prioritization results we have extended our previous work in this study by applying four different strategies to prioritize candidate genes based on network analysis of differential expression using distinct machine learning approaches to determine whether a gene is surrounded by highly differentially expressed genes in a functional association or proteinprotein interaction network. Here, we have mainly focused on further performance improvement and determination of an appropriate neighborhood for network propagation of differential expression analysis by considering and benchmarking many variables occurring here in the presented ranking strategies. We have further compared our prioritization results with a standard procedure in genetics that ranks candidate genes based solely on their differential expression levels.

First, we propose an alternative to our previous idea of defining a notion of distance using the Laplacian exponential diffusion kernel. Instead of aggregating the differential expression of neighbors weighted as a function of distance, we have smoothed a candidate gene's differential expression levels through kernel ridge regression. We name this strategy Kernel Ridge Regression Ranking.

Second, we have applied the Heat Kernel Diffusion Ranking, introduced by Chung and Yau (1999) [10], to our problem of disease candidate gene prioritization.

Third, we have carried out network diffusion by applying the Arnoldi algorithm based on a Kyrlov Space method as presented in [11]. We name this strategy Arnoldi Diffusion Ranking.

Fourth, we have ranked the candidate genes by combining their differential expression levels with the average of the differential expression levels among their direct neighbors in a functional association or proteinprotein interaction network, which we name Direct Neighborhood Ranking. This straightforward approach for scoring candidates is presented here for comparison purposes as a naïve strategy for network analysis of differential expression.

We have benchmarked these four strategies on 40 publicly available data sets originated from Affymetrix chips on which mice with (simple) knockout genes were 
tested against controls. The raw cel files were downloaded from GEO [12]. For each data set we have computed the differential expression levels for each gene in our network based on the expression in the knockout experiment versus the expression in the control (see Methods).

Since we were seeking for a suitable interaction network for our application, we have considered two different types of networks: functional association networks and protein-protein interaction (PPI) networks. As a functional association network, we have used two different STRING releases (version 7.1 [13] and version 8.2 [14]), which include associations and physical interactions coming from heterogeneous databases. As PPI networks, we have used BioGRID [15] and I2 D [16]. By using different networks in our ranking strategies, we evaluated their suitability as an interaction network for our application, and to what extent performance is influenced by the characteristics of the underlying network.

In the field of cancer, some methods for network analysis are available for gene expression towards identification of expression signatures or of dysregulated subnetworks as biomarkers. However, the task we address is that of prioritization of disease causing genes in constitutional genetic disorders using expression data. That problem is different from that of signature identification in cancer because it focuses on the ranking of candidate genes instead of the identification of subnetworks (see for example [17-19]). There is no established method to tackle the specific problem of candidate gene prioritization in constitutional disorders from expression data.

\section{Results}

In this section, we have evaluated the gene prioritization results obtained using each of the presented strategies, focusing on performance improvement. We aim to assess whether machine learning approaches based on random walks can outperform a standard procedure in genetics, the Simple Expression Ranking (see for example [20-22]).

The previous described methods require several parameters that we have tuned in order to obtain stable ranking results. Additionally, we combine several preprocessing techniques, expression measures and networks:

\section{(1) Preprocessing}

Gene expression data was preprocessed using three different techniques: MAS5, RMA and GCRMA (for details see Methods section).

(2) Expression measure

We have computed three different expression measures: $\log 2$ ratio, the test statistic (derived from CyberT [23], and the significant log2 ratio (p-value derived from CyberT).

(3) Network

Four different mouse networks were selected for diffusion: two versions of the functional association network obtained from STRING (versions 7.1 and 8.2), a PPI network obtained from BioGRID (version 2.0.61), and a PPI network obtained from I2 D (version 1.72).

All strategies were applied to these four networks. The Similarity Network for the Kernel Ridge Regression Ranking was obtained using the Laplacian Exponential Diffusion kernel $(\alpha=0.5)$, the Regularized Commute Time kernel ( $\alpha=0.90$ and $\alpha=0.95$ ), and the Regularized Laplacian Diffusion kernel ( $\alpha=1$ and $\alpha=2$ ), see equations (1)-(3). For the Heat Kernel Diffusion Ranking we have applied discrete approximation of the Heat Kernel rank approach [24], see equation (9). For the Arnoldi Diffusion Ranking we have applied Arnoldi approximation [11], see equation (11). Finally, for the Direct Neighborhood Ranking we have used the network directly to capture a neighborhood for a candidate gene, see equation (13).

(4) Methods and parameter setting Kernel Ridge Regression Ranking, Heat Kernel Diffusion Ranking, and Arnoldi Diffusion Ranking require a value for the diffusion rate $\alpha$. We have chosen this parameter to be 0.5 for all strategies. Kernel Ridge Regression Ranking requires two parameters, $\lambda$, for which we have chosen five different values $\left\{10^{-2}, 10^{-1}, 10^{0}, 10^{1}, 10^{2}\right\}$, and $n n$, the maximum number of neighbors, for which we have chosen three values: 30, 50, and 100 .

Heat Kernel Diffusion Ranking requires one parameter, $m$, defining the number of iterations, i.e., the number of steps in the random walk through the network. Arnoldi Diffusion Ranking requires one parameter, $m$, defining the number of iterations for obtaining an approximation of the network diffusion using the Arnoldi algorithm.

Direct Neighborhood Ranking requires a weight, $a$, for which we chose a value of 0.5 , so that both the expression of the candidate itself and its average surrounding expression in the network were weighted equally. As potential values for $\varepsilon$, we have chosen $0.15,0.4$ and 0.7 (regarding the confidence scores in STRING).

\section{Validation}

The parameter settings were tuned to obtain stable ranking results for the benchmark data, indicated by the rank of the knockout gene. We have computed ROC 
curves and the corresponding AUC values and counted the number of knockout genes ranked in the top $10 \%$ for all ranking lists using all parameter settings. A description of the parameter tuning procedure follows. In this step, we used the STRING network (v.7.1) in all the presented strategies. After the determination of the optimal parameters, we have explored alternative kernels in the Kernel Ridge Regression Ranking, different preference vectors in the Heat Kernel Diffusion Ranking, and distinct networks in all strategies using the determined parameter settings.

\section{Parameter Tuning}

Table S1a (see additional file 1) illustrates the ranking results obtained by applying the Kernel Ridge Regression Ranking using different values for $\lambda$ and $n n$ and the Laplacian Exponential Diffusion Kernel with $\alpha=0.5$ using the STRING network (v.7.1). Based on the results presented we have chosen $\lambda$ to be 1 and $n n$ to be 50 since these values lead to the most stable ranking results in comparison to other values, considering the AUC and the number of top ranked knockout genes.

Table S1c (see additional file 1) illustrates the ranking results obtained by applying the Heat Kernel Diffusion Ranking using distinct number of steps in the random walk through the STRING network (v.7.1). These results clearly show that the method is able to generate a reliable ranking in a reduced number of steps, i.e. $m=2$.

Table S1f (see additional file 1) illustrates the ranking results obtained by applying the Arnoldi Diffusion Ranking using distinct number of iterations in the Arnoldi approximation and the STRING network (v.7.1). The results clearly show that the method is able to produce a reliable ranking using only 2 iterations, as in the case of the Heat Kernel Diffusion Ranking, i.e. $m=2$.

Table S1e (see additional file 1) illustrates the ranking results obtained by applying the Direct Neighborhood Ranking using the STRING network (v.7.1) and $a=0.5$ with distinct thresholds $\varepsilon$. Results show a decrease in performance with the increase of $\varepsilon$, due to information loss (many associations are then missing from the network), i.e. $\varepsilon=0.15$.

Table 1 shows the overall results of the four strategies including their best performing parameters, and using two different releases of the STRING network (version 7.1 and version 8.2).

\section{Kernels in Kernel Ridge Regression Ranking}

The Kernel Ridge Regression Ranking is based on a kernel matrix derived from the STRING network or the PPI network from BioGRID or I2 D, as shown in equations (1)-(4). As discussed in the Methods section, several kernel matrices are available in the literature. In this study, we have assessed which of the three considered kernels performs best in our application. Table S1b (see additional file 1) shows the results based on the Laplacian Exponential Diffusion Kernel, Regularized Laplacian Kernel and Regularized Commute time Kernel with different parameters. It stands out that the Laplacian Exponential Diffusion Kernel with $\alpha=0.5$ performs better than the other kernels. Thus, in our study, the Laplacian Exponential Diffusion kernel with $\alpha=0.5$ will be used as the kernel matrix in the Kernel Ridge Regression Ranking.

\section{Preference vector initialization in Heat Kernel Diffusion Ranking}

Francisco et al. (2009) suggested initializing the preference vector $p_{0}$ with binary values [25]: the seed genes known to be involved in the disease would be set to 1 , as all other genes in the vector would be set to 0 . In order to assess the contribution of the gene expression levels to the ranking, we have compared four different scenarios: first, only the candidate genes were initialized with 1 and the remaining genes with 0 (same procedure as in [25]), independent of their expression value. Second, we have only initialized the candidate genes with their differential expression levels obtained in the experiment and the remaining genes with 0 . Third, we have filled the preference vector $p_{0}$ with all the expression values available in the experiment. Fourth, we have initialized all genes that are differentially expressed in the experiment with 1 , all other genes with 0 , thus we have made no difference between genes that were highly or weakly differentially expressed. Table S1d (see additional file 1) shows the result of these four scenarios for initializing the preference vector.

It can be seen that by using only binary values (scenario 1), the Heat Kernel Diffusion Ranking performs poorly (AUC $=61.2 \%$ with only 5 knockout genes in the top $10 \%$ ). However, using expression values instead of binary ones, the method performs better. This shows that the contribution of expression levels coming from a disease or knockout related microarray experiment is significant. Furthermore, if all available expression values are added to the preference vector (scenario 3), the results were further improved against the ones obtained by initializing the preference vector with the expression levels of the candidate genes only (scenario 2). However, if the expression values are replaced by binary ones (scenario 4), the results are slightly worse than considering differential expression values. By considering all available expression values (scenario 3), the ranking obtained between 31 and 32 top 10\% ranked knockout genes with an AUC of between $88.9 \%$ and $90.7 \%$ for the $\log 2$ ratio or the test statistic as the expression measure, independent of the preprocessing technique. In subsequent steps, we use all available expression data for initializing 
Table 1 Overview of prioritization results

\begin{tabular}{|c|c|c|c|c|c|c|c|c|}
\hline & & & & STRI & v. 7.1 & & STRI & v. 8.2 \\
\hline & & & top 10 & top 20 & AUC & top 10 & top 20 & AUC \\
\hline & Standard genetic procedure: Simple Expr & ion Ranking & 20 & 25 & 0.801 & 20 & 25 & 0.801 \\
\hline & Direct Neighborhood Ranking & $\log 2$ ratio & 27 & 31 & 0.859 & 12 & 23 & 0.747 \\
\hline & $\varepsilon>0.15, a=0.5$ & sign. $\log 2$ ratio & 28 & 31 & 0.880 & 12 & 24 & 0.760 \\
\hline & & test statistic & 29 & 30 & 0.856 & 13 & 22 & 0.738 \\
\hline & Kernel Ridge Regression Ranking & $\log 2$ ratio & 23 & 29 & 0.809 & 14 & 26 & 0.759 \\
\hline & $\lambda=1, n n=50, K_{L E D}, \alpha=0.5$ & sign. $\log 2$ ratio & 27 & 32 & 0.868 & 17 & 25 & 0.817 \\
\hline RRMA & test statistic & 20 & 26 & 0.771 & 15 & 20 & 0.691 & \\
\hline & Heat Kernel Diffusion Ranking & $\log 2$ ratio & 32 & 34 & 0.900 & 32 & 33 & 0.913 \\
\hline & all expression values for $p_{0}, m=2, \alpha=0.5$ & sign. $\log 2$ ratio & 31 & 34 & 0.910 & 31 & 35 & 0.923 \\
\hline & & test statistic & 32 & 34 & 0.901 & 32 & 34 & 0.911 \\
\hline & Arnoldi Diffusion Ranking & $\log 2$ ratio & 27 & 31 & 0.857 & 27 & 29 & 0.851 \\
\hline & $m=2, \alpha=0.5$ & sign. $\log 2$ ratio & 28 & 31 & 0.885 & 28 & 31 & 0.873 \\
\hline & & test statistic & 28 & 30 & 0.855 & 28 & 29 & 0.844 \\
\hline & Standard genetic procedure: Simple Expr & ion Ranking & 18 & 24 & 0.777 & 18 & 24 & 0.777 \\
\hline & Direct Neighborhood Ranking & $\log 2$ ratio & 27 & 31 & 0.874 & 12 & 23 & 0.761 \\
\hline & $\varepsilon>0.15, a=0.5$ & sign. $\log 2$ ratio & 25 & 28 & 0.855 & 11 & 22 & 0.736 \\
\hline & & test statistic & 27 & 31 & 0.863 & 12 & 24 & 0.750 \\
\hline & Kernel Ridge Regression Ranking & $\log 2$ ratio & 21 & 28 & 0.769 & 17 & 24 & 0.756 \\
\hline & $\lambda=1, n n=50, K_{L E D}, \alpha=0.5$ & sign. $\log 2$ ratio & 27 & 31 & 0.835 & 20 & 26 & 0.796 \\
\hline GCRMA & & test statistic & 19 & 22 & 0.745 & 16 & 23 & 0.744 \\
\hline & Heat Kernel Diffusion Ranking & $\log 2$ ratio & 31 & 33 & 0.905 & 32 & 34 & 0.914 \\
\hline & all expression values for $p_{0}, m=2, \alpha=0.5$ & sign. $\log 2$ ratio & 28 & 33 & 0.889 & 29 & 34 & 0.907 \\
\hline & & test statistic & 32 & 33 & 0.895 & 32 & 35 & 0.913 \\
\hline & Arnoldi Diffusion Ranking & $\log 2$ ratio & 27 & 32 & 0.875 & 26 & 31 & 0.874 \\
\hline & $m=2, \alpha=0.5$ & sign. $\log 2$ ratio & 25 & 29 & 0.860 & 25 & 28 & 0.852 \\
\hline & & test statistic & 27 & 31 & 0.865 & 28 & 31 & 0.862 \\
\hline & Standard genetic procedure: Simple Expr & ion Ranking & 24 & 28 & 0.837 & 24 & 28 & 0.837 \\
\hline & Direct Neighborhood Ranking & $\log 2$ ratio & 23 & 27 & 0.846 & 9 & 20 & 0.743 \\
\hline & $\varepsilon>0.15, a=0.5$ & sign. $\log 2$ ratio & 25 & 28 & 0.844 & 11 & 22 & 0.729 \\
\hline & & test statistic & 27 & 30 & 0.855 & 12 & 24 & 0.745 \\
\hline & Kernel Ridge Regression Ranking & $\log 2$ ratio & 18 & 24 & 0.736 & 17 & 24 & 0.766 \\
\hline & $\lambda=1, n n=50, K_{L E D}, \alpha=0.5$ & sign. $\log 2$ ratio & 23 & 29 & 0.834 & 17 & 22 & 0.755 \\
\hline MAS5 & & test statistic & 13 & 18 & 0.790 & 16 & 24 & 0.790 \\
\hline & Heat Kernel Diffusion Ranking & $\log 2$ ratio & 26 & 32 & 0.877 & 28 & 34 & 0.890 \\
\hline & all expression values for $p_{0}, m=2, \alpha=0.5$ & sign. $\log 2$ ratio & 26 & 31 & 0.877 & 26 & 34 & 0.899 \\
\hline & & test statistic & 32 & 32 & 0.890 & 31 & 34 & 0.904 \\
\hline & Arnoldi Diffusion Ranking & $\log 2$ ratio & 25 & 27 & 0.849 & 24 & 27 & 0.851 \\
\hline & $m=2, \alpha=0.5$ & sign. $\log 2$ ratio & 25 & 29 & 0.853 & 25 & 27 & 0.847 \\
\hline & & test statistic & 26 & 30 & 0.858 & 27 & 29 & 0.850 \\
\hline
\end{tabular}

The results are based on optimized parameter settings for all presented strategies using different STRING networks. Note that the results of the Simple Expression Ranking do not depend on the network because they are only using differential expression levels to rank the candidate genes.

the preference vector in the Heat Kernel Diffusion Ranking.

\section{Error Reduction}

Table 1 shows that the Simple Expression Ranking performs well: using MAS5 preprocessed data we have obtained an AUC of $83.7 \%$. However, by applying the presented machine learning strategies based on random walks, the results could be further improved.

Results of the Heat Kernel Diffusion Ranking using RMA preprocessed data have obtained an AUC value of 91\% and $92.3 \%$ using the STRING networks v7.1 and v8.2, respectively, and the significant $\log 2$ ratio as expression measure. This presents an error reduction of 
$52.8 \%$ relative to the Simple Expression Ranking for the STRING network v8.2 and $44.8 \%$ for the STRING network v7.1. Regarding the number of knockout genes, the Heat Kernel Diffusion Ranking has ranked a maximum of 32 knockout genes in the top $10 \%$ for both STRING network releases using the test statistic as expression measure, independent of the preprocessing technique. The Simple Expression Ranking could rank at most 24 knockout genes in the top $10 \%$ for MAS5 preprocessed data, which again presents an error reduction of $50 \%$ using the Heat Kernel Diffusion Ranking.

The Kernel Ridge Regression Ranking strategy could outperform the Simple Expression Ranking only for RMA using the significant $\log 2$ ratio as expression measure and STRING network v7.1. The corresponding error reduction was of $19 \%$ regarding the AUC $(86.8 \%)$ and the number of knockout genes (27) relative to the Simple Expression Ranking using MAS5. For STRING v8.2 and all other settings the Kernel Ridge Regression Ranking could not outperform the Simple Expression Ranking.

The Arnoldi Diffusion Ranking strategy could outperform the Simple Expression Ranking for RMA preprocessed data using the significant $\log 2$ ratio or test statistic as expression measure and STRING v7.1 or for GCRMA preprocessed data using the $\log 2$ ratio as expression measure and STRING v8.2. The error could be reduced by $29.4 \%$ in terms of the AUC for STRING v7.1 (88.5\%) and by $22.7 \%$ for STRING v8.2 (87.4\%). Regarding the number of top ranked knockout genes, we could reduce the error for both networks by $25 \%$ (28 top ranked knockout genes) relative to the Simple Expression Ranking. For some other settings this strategy could outperform the Simple Expression Ranking as well.

The Direct Neighborhood Ranking strategy could outperform the Simple Expression Ranking for RMA preprocessed data using the significant $\log 2$ ratio or test statistic as expression measure and the STRING network v7.1. The error could be reduced by $26.4 \%$ in terms of the AUC (88\%) and by $31 \%$ in terms of the number of knockout genes (29) relative to the Simple Expression Ranking using MAS5 preprocessed data. For some other settings this strategy could outperform the Simple Expression Ranking as well. However, for STRING v8.2 the Direct Neighborhood Ranking could not outperform the Simple Expression Ranking.

As a consequence, the Heat Kernel Diffusion Ranking shows the largest error reduction relative to the Simple Expression Ranking considering both the AUC values and the number of top ranked knockout genes. Furthermore, these results were achieved independent of the preprocessing technique and for both STRING network releases.

\section{Analysis of dependency on expression levels}

We have further analyzed the influence of the expression levels of the candidate genes in the performance of the four presented ranking strategies. For this purpose, we have set the expression levels of the knockout genes to $O$ and compared with the original results. Table S2 shows that none of the strategies achieves a comparable level of performance if the expression level of the knockout gene is set to 0 . Therefore it is reasonable to assume that the performance of all four strategies in this study is highly dependent on integration of the expression levels of the knockout gene.

\section{Comparison of different networks: functional association networks vs. protein-protein interaction networks}

As shown in the Methods Section, the mouse PPI network originated from BioGRID contains substantially less information then both mouse STRING networks. Nevertheless, we have analyzed to what extent the gene prioritization methods depend on the choice of the network and how sparsity is influencing the results.

Table 1 illustrates a direct comparison between both STRING releases using the four gene prioritization strategies. It shows that the performance of both, the Kernel Ridge Regression Ranking and Direct Neighborhood Ranking, using STRING v7.1 is better than using STRING v8.2. By updating the STRING network from version 7.1 to 8.2 , the error was increased by approx. $30 \%-50 \%$ in comparison to version 7.1 , depending on the parameter setting. However, the performance for the Heat Kernel Diffusion Ranking and Arnoldi Diffusion Ranking applying both STRING releases are comparable, even though the releases differ substantially from each other. This demonstrates that these two strategies are not as dependent on the choice of the network as the other two. On the other hand, by updating the STRING network from version 7.1 to 8.2 the performance could not be improved further using the Heat Kernel Diffusion Ranking or Arnoldi Diffusion Ranking as expected.

Figure 1 shows the ROC curves of the performance of the presented strategies for gene prioritization using RMA preprocessed data and the significant log2 ratio as the expression measure for both STRING network releases in comparison to the Simple Expression Ranking using MAS5 preprocessed data. The reason for choosing RMA as the preprocessing technique and the significant $\log 2$ ratio as the expression measure is that this setting leads generally to the most stable and reliable result in our study (see Table 1).

Using the mouse PPI network obtained from the BioGRID database, we could not obtain good quality ranking results for our benchmark. Out of the 40 data sets, only six could be used on the PPI network because the knockout genes of the other 34 data sets were absent 


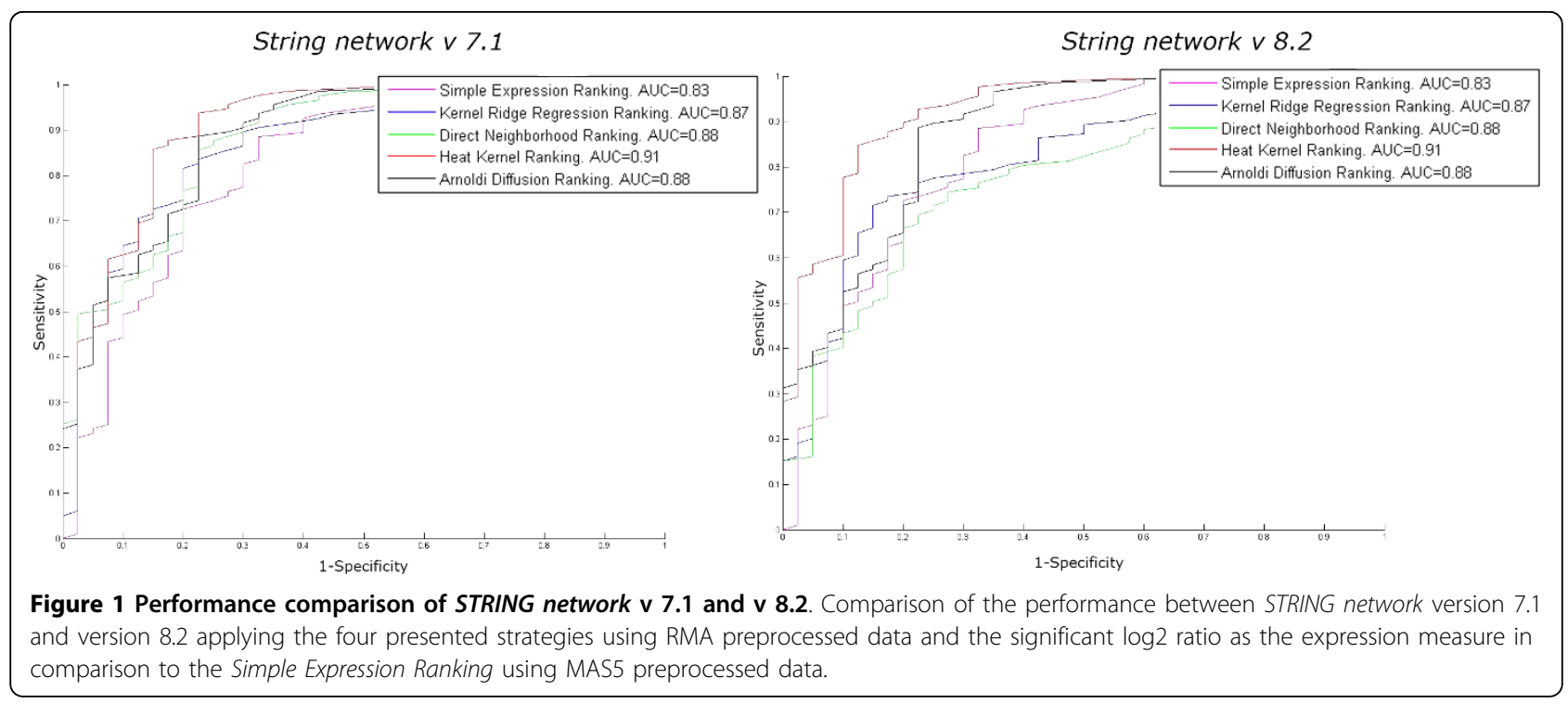

from the PPI network (this network is very sparse, see Table 2). For validating the performance of the strategies, we have used 100 candidate genes that were ranked for each data set. However, using this PPI network, only 4-10 of the candidate genes were found in the network (data not shown). Therefore, we could not perform a meaningful comparison between the functional association network from STRING and the PPI network from BioGRID. Thus, we can draw the conclusion that a sparse network, like the BioGRID mouse network, is not suitable for our application.

The mouse PPI network from the I2 D database is a higher-coverage PPI network (see Table 2). However, like for the BioGRID mouse network, the I2 D mouse network is missing knockout genes from the benchmark. Only 35 out of 40 data sets could be used on the PPI network because the others were incomplete (data not shown). For this reason we have extended the network by five additional nodes which are isolated from the other nodes in the networks, since the database does not provide information about interactions between these nodes and the others in the network. Table S3 (see additional file 1) shows that by using the I2 D network the results coming from the Heat Kernel Diffusion Ranking and the Arnoldi Diffusion Ranking were only slightly better than those obtained by the Simple Expression Ranking, for the other ranking strategies the I2 D network performed worse in terms of the AUC value and top ranked knockout genes. Comparing with the STRING network, our results have shown that a functional association network like the STRING network performs better in our application than a PPI network.

\section{Comparison of different expression measures and preprocessing techniques}

In this study, we have evaluated not only different networks applied on the four presented strategies, but also different expression measures in combination with the preprocessing techniques applied by the strategies. Table 1 illustrates that in our application RMA best results correlates with the significant $\log 2$ ratio as expression measure, GCRMA with the $\log 2$ ratio, and MAS5 with test statics. In terms of the presented prioritization strategies, Table 1 shows that the Kernel Ridge Regression best results correlate with the significant $\log 2$ ratio as expression measure, and the Heat Kernel Diffusion Ranking with test statistic and significant $\log 2$ ratio.

These results show that there is no clear conclusion to draw in terms of identifying the best performing preprocessing technique or expression measure. Finding the best performing preprocessing technique is a challenge and has been widely discussed in the literature, such as [26-29].

\section{Table 2 Overview of the global network properties of the underlying networks}

\begin{tabular}{lrrr}
\hline Database (mouse) & Number of Genes & Number of Interactions & Average Node Degree \\
\hline STRING v7.1 & 16,566 & 820,177 & 49.5 \\
STRING v8.2 & 24,442 & $1,405,375$ & 57.5 \\
BioGRID v2.0.61 & 1,417 & 2,026 & 2.5 \\
I2 D v1.72 & 10,867 & 79,088 & 10.6 \\
\hline
\end{tabular}




\section{Discussion}

As we have introduced in this study, there are several methods in the literature to prioritize candidate genes. Among these approaches, there are methods requiring knowledge about disease-gene association, whereas others do not have this precondition. However, these methods will be ineffective if no knowledge is available for a specific disease. In our previous study, we introduced an approach to overcome this limitation by replacing this knowledge by disease-specific experimental data [9].

The purpose of this study was to further improve the performance of the prioritization results and the determination of an appropriate neighborhood for network analysis which has been a major issue in our previous work. We have extended our previous work extensively by suggesting four strategies to prioritize candidate genes based on distinct machine learning approaches to determine whether a gene is surrounded by highly differentially expressed genes in a network, by considering variations in parameter settings, usage of different kernel functions, preprocessing techniques and expression measurements. All prioritization results coming from these different settings were benchmarked on 40 mouse knockout data sets computing AUC values and the number of knockout genes ranked in the top $10 \%$. The performance was assessed against results obtained using a standard procedure in genetics that ranks candidate genes based solely on their differential expression levels (Simple Expression Ranking) that was clearly outperformed by the here presented ranking strategies in terms of AUC values and ranking positions of knockout genes (see Results section).

We have used three distinct random walk based strategies plus one naïve strategy without network diffusion but a direct neighborhood analysis. The random walk strategies all base on the Exponential Diffusion Kernel, although the methodology is distinct: the Kernel Ridge Regression Ranking computes the (convergent) kernel to solve a regression problem, the Heat Kernel Diffusion Ranking computes an iterative diffusion with only 2 steps over the network and combines it with expression data coming from the experiment which is the Heat Kernel rank, and the Arnoldi Diffusion Ranking combines an approximation of the kernel with expression data. It has to be noted that the convergence in the Heat Kernel Diffusion Ranking and in the Arnoldi Diffusion Ranking has been measured in terms of ranking results and not in terms of kernel assimilation which makes it not comparable to the kernel matrix computation resulting from the Kernel Ridge Regression Ranking.

Although we have considered and benchmarked many variables occurring in our ranking strategies, there are still a few that influence the performance of our prioritization strategies.

First, the quality and coverage of the network around the actual disease gene will be a strict bottleneck as we already analyzed in [9]. For example, an isolated gene with no edges in the network can not be effectively prioritized by our methods. We have used the STRING network based on the assumption that a network, integrating multiple heterogeneous data sources, is more complete and robust and therefore less prone to the problems caused by sparsity. Further, we must note that extending the data sources from version 7 to 8 has resulted in a larger network with good coverage. Errors in the network might cause both false negatives and false positives.

Continuing improvements in the quality of protein association networks are expected to contribute to increased effectiveness of the proposed method which could be demonstrated by comparing the two releases STRING v7.1 and STRING v8.2. In the latter release the performance could be increased further from $91 \%$ to 92.3\% using the Heat Kernel Diffusion Ranking, although the performances obtained by using both releases are not as significant as by using the Kernel Ridge Regression Ranking and Direct Neighborhood Ranking. The performance for the Kernel Ridge Regression Ranking and Direct Neighborhood Ranking using the STRING network v7.1 was better than using the STRING network v8.2. By updating the STRING network from version 7.1 to 8.2, the error was increased by approx. 30\%-50\%, dependent of the setting.

Worsening in the performance using the updated version of the STRING network in the Direct Neighborhood Ranking could be explained by the fact that we could observe more neighbors for the candidate genes so that a few highly differentially expressed genes lost their influence in comparison to version 7.1 in which fewer neighbors were detected. For the Kernel Ridge Regression Ranking, however, the similarity matrices of both STRING releases differ substantially from each other and cannot be compared directly since there is only a small overlap between both STRING networks.

To evaluate to what extent the coverage of the interaction network influences our method, we have further applied 2 distinct PPI networks of different coverage. Using the BioGRID network, results have shown that this network was too sparse for our application because most of the knockout genes from the data sets and candidate genes to be ranked in the underlying study were absent from the network (data not shown). Using the I2 D network, results have shown that we could slightly outperform our Baseline, the Simple Expression Ranking. Nevertheless, the STRING network is more densely 
connected and contains more genes (see Table 2), which led to a better performance for the ranking results.

Like in the BioGRID network, some knockout genes were also absent from the I2 D network. Therefore we extended the network by these nodes, but with no interaction between them and the other nodes in the network. Our results have shown that some of the isolated genes could be ranked highly (data not shown) despite their missing interactions because the ranking strategies also consider the candidate genes' expression levels. As long as an isolated gene has a large differential expression level, it can be ranked highly. However, this is not a guarantee to detect this gene among the top ranked genes because a highly differentially expressed neighborhood will still dominate. Therefore an isolated gene cannot be reliably prioritized by our ranking strategies.

Nevertheless, the ranking strategies using the STRING network have performed better than using the I2 D network, probably because of the difference in coverage. One may argue that it is not appropriate to compare a PPI network with a functional association network because the latter is including associations apart from physical interactions, but we are seeking for a suitable and high-coverage interaction network for our application that leads to reliable ranking results of candidate genes, regardless of the nature of the associations.

In the literature, several databases can be found to construct an interaction network that can be categorized in terms of types and scope of data sources, types of interactions, or range of details on protein interactions [30]. Comprehensive protein interaction databases, such as BioGRID [15], DIP [31], BIND [32], IntAct [33], MINT [34], I2 D [16], iRefIndex [35] or STRING [13,14] collect physical interactions or functional associations between proteins, integrating distinct information sources about protein interactions coming from high-throughput experimental data, structural data, manual curation, or functional predictions. Specialized protein interaction databases, such as MIPS [36] or HPRD [37] collect (manually) curated interactions from yeast or human, respectively. For this study, the BioGRID database was used to represent a (sparse) PPI network containing only high-throughput experimental data, the I2 D database was used to represent a high-coverage PPI network integrating different information sources, and STRING was used to represent a high-coverage functional association network combining associations and physical interactions coming from distinct data sources.

In Nitsch et al. (2009) we adopted the hypothesis from Köhler et al. (2008) that global network-similarity measures capture relationships between disease proteins better than algorithms based on direct interactions [2,9]. The latter was considered in the Direct Neighborhood Ranking strategy which performed worse than the Heat
Kernel Diffusion Ranking leading to a global diffusion measure. Since we have proposed the Direct Neighborhood Ranking as a naïve method for comparison purposes, we have already expected not to outperform the other three random walk based strategies because of its systematic bias favoring highly connected genes in the network (which we discuss later).

Second, the integration of expression data proved to have a significant influence on the performance of the prioritization strategies which is supported by the results using the mouse knockout experiments data. By using mouse knockout data sets, we could guarantee to use disease relevant tissue which is an important issue in identifying disease related genes (disease genes might not be expressed in other tissues). We have further analyzed the dependency of the ranking performance on the preprocessing of expression data. In the literature there are many controversial discussions about the performance of these, including MAS5, RMA, and GCRMA, and the community cannot harmonize (see for example [26-29]). For this reason we have applied MAS5, RMA, and GCRMA on the benchmark data sets leading to different ranking results, as we would have expected.

In the Results section we have claimed that we could not draw a clear conclusion in terms of identifying the best performing preprocessing technique or expression measure. Nevertheless, the best results were achieved using RMA preprocessed data in comparison to the other preprocessing techniques. However, in other applications alternative techniques might perform better. The same matters for finding an appropriate expression measure. In the literature there are many measures, from which we have chosen the $\log 2$ ratio, the significant $\log 2$ ratio, and test statistic derived from CyberT. By analyzing their performance in our application, we could not claim one measure to be outperforming another, however, the significant $\log 2$ ratio led to the most stable results over the majority of the settings.

The quality of the experimental data has also an important impact on the performance of the prioritization strategies. By using expression data that was either incomplete or of poor quality, the prioritization strategies could not lead to reliable results. The sensitivity of the different scoring strategies or the underlying networks to this effect may vary (for example, PPI networks have been reported to be more sensitive to this effect). This issue may come up when applying the presented method to real genetic disorders in human. In our previous study, we could already detect four known disease genes in human using public available expression data of good quality [9]. Therefore, the bottleneck of our method is again the sparseness and quality of experimental data. 
Third, the choice of appropriate parameters for the presented strategies has a large influence on the performance. Therefore we had several parameters and settings to optimize. By using the optimal parameters and settings, we have obtained stable and robust results for the prioritization problem. All presented strategies have outperformed our Baseline, the Simple Expression Ranking - as we have expected - for certain settings. The Heat Kernel Diffusion Ranking has shown the largest error reduction relative to the Simple Expression Ranking in terms of AUC values and number of top ranked knockout genes, independent of the preprocessing technique and for both STRING network releases. Our results show that the Simple Expression Ranking is performing well for MAS5 preprocessed data. However, the Heat Kernel Diffusion Ranking on RMA preprocessed data could achieve and error reduction of $52.8 \%$ for the STRING network v8.2 and $44.8 \%$ for the STRING network v7.1.

Fourth, the fact that a disease related gene may have only a weakly differentially expressed neighborhood negatively affects the prioritization methods. In this case, our hypothesis of observing a strongly differentially expressed neighborhood for disease related genes can fail. By introducing a better experimental design that can trigger a disease pathway more reliably in which the effect is more focused around the disease gene, would overcome this limitation because in our approach we do not consider pathways but only neighbors that are surrounding a gene in the network. In this case the Simple Expression Ranking can perform better as long as the disease gene is highly differentially expressed since this naïve method does not depend on expressed pathways or neighborhoods.

Fifth, a systematic bias using a biological network favoring highly connected genes can be observed in our method which leads to sensitivity to skewed degree distributions. This bias is clearly a limitation of our method and can be addressed through proper randomization. By randomizing signals across the network, the bias caused by the higher connectivity (or higher total weights) can be removed since highly connected nodes will have a higher baseline signal across the randomizations. We are currently investigating this issue.

\section{Conclusion}

In this study, we have extended extensively our previous work by applying machine learning approaches based on random walk models to determine whether a gene's neighborhood is highly differentially expressed. We have explored three different random walk based strategies plus one naïve strategy based on a direct neighborhood analysis. These four network-based prioritization strategies for scoring candidate genes based on their differentially expressed neighborhood were benchmarked on 40 publicly available knockout experiments in mice. Performance was assessed against results obtained using a standard procedure in genetics that ranks candidate genes based solely on their differential expression levels (Simple Expression Ranking). Results showed that our strategies could outperform this standard procedure and that the best results were obtained using the Heat Kernel Diffusion Ranking leading to an average ranking position of 8 out of 100 genes, an AUC value of $92.3 \%$ and an error reduction of $52.8 \%$ relative to the standard procedure approach which ranked the knockout gene in average at position 17 with an AUC value of $83.7 \%$. Thus, we could identify promising candidate genes using networkbased machine learning approaches even if no knowledge is available about the disease or phenotype.

\section{Methods \\ Benchmark data}

The benchmark for this study consists of 40 publicly available data sets originated from Affymetrix chips on which mice with knockout genes were tested against controls. The raw cel files were downloaded from GEO [12]. Table 3 shows all data sets used in our benchmark.

\section{Preprocessing}

We have preprocessed the gene expression data using three different preprocessing techniques: MAS5 (Affymetrix Microarray Suite 5.0) [38], RMA [39], and GCRMA [40]. Moreover, we have assessed their individual contribution to results seeking the best performance in a number of validation tests.

We have implemented the preprocessing techniques in $\mathrm{R}$ using the BioConductor package which includes a large number of meta-data packages available that are oriented towards different types of microarrays. Affy is the most common library that is used for data processing and visualization of Affymetrix GeneChip measurements [41].

\section{Differential expression measures}

After the preprocessing step we have computed the differential expression level for each gene in the network based on the expression in the knockout experiment versus the expression in the control for each data set.

We have used three different measures of differential expression:

- $\log 2$ ratio that is defined as $\log _{2}\left(\frac{\text { mutant }}{\text { control }}\right)$.

- Significant $\log 2$ ratio: only significant $\log 2$ ratios (pvalue $<0.05$ ) are considered, otherwise they are set to 0 . The pvalue was computed by CyberT.

- Test statistic: computed from CyberT. 
Table 3 The benchmark data

\begin{tabular}{|c|c|c|c|c|c|}
\hline & Gene Name & GEO accession number & & Gene Name & GEO accession number \\
\hline 1 & Abca1 & GSE5496 & 21 & Mbnl1 & GSE14691 \\
\hline 2 & Btk & GSE2826 & 22 & Mst1r, Ron & GSE16629 \\
\hline 3 & Cav1 & GSE10849 & 23 & MyD88 & GSE6688 \\
\hline 4 & Cav3 & GSE10848 & 24 & Nos3, eNos & GSE1988 \\
\hline 5 & Cftr & GSE5715 & 25 & Phgdh & GSE8555 \\
\hline 6 & Clen1 & GSE14691 & 26 & Pmp22 & GSE1947 \\
\hline 7 & Cnr1 & GSE7694 & 27 & $\operatorname{PPAR} \alpha$ & GSE6864 \\
\hline 8 & Emd & GSE5304 & 28 & Prkag3, AMPK G3 & GSE4065 \\
\hline 9 & Epas1, Hif-2 & GSE16067 & 29 & Pthlh, Pthrp & GSE17654 \\
\hline 10 & Esrra & GSE7196 & 30 & Rab3a & GSE6527 \\
\hline 11 & Gap43 & GSE12687 & 31 & RasGrf1 & GSE8425 \\
\hline 12 & Gnmt & GSE9809 & 32 & $\mathrm{Rbm} 15$ & GSE12628 \\
\hline 13 & Hdac1 & GSE5583 & 33 & Runx & GSE4911 \\
\hline 14 & Hdac2 & GSE6770 & 34 & Scd1 & GSE2926 \\
\hline 15 & $\mathrm{Hsf4}$ & GSE12415 & 35 & Slc26a4 & GSE10587 \\
\hline 16 & Hspa1A, Hsp70.1 & GSE11120 & 36 & Srf & GSE13333 \\
\hline 17 & 116 & GSE411 & 37 & Tgm2 & GSE10285 \\
\hline 18 & Lhx1, Lim1 & GSE4230 & 38 & Zc3h12a & GSE14891 \\
\hline 19 & Lhx8 & GSE11897 & 39 & Zfp36, Tpp & GSE5324 \\
\hline 20 & Lmna & GSE5304 & 40 & $Z f x$ & GSE7069 \\
\hline
\end{tabular}

The benchmark consists of 40 publicly available data sets originated from Affymetrix chips on which mice with (simple) knockout genes were tested against controls.

CyberT [23] employs statistical analyses based on simple t-tests that use the observed variance of replicate gene measurements across replicate experiments, or regularized $t$-tests that use a Bayesian estimate of the variance among gene measurements within an experiment.

\section{Network}

A functional protein association network is an undirected graph with proteins as nodes and associations as edges. Whenever there is a functional association between two proteins, an edge will be set between the corresponding nodes in the graph. The weights of the edges represent a confidence value on the evidence of such an association. The functional protein association network for mouse was obtained from STRING [13], a database of known and predicted protein-protein associations derived from heterogeneous data sources and different organisms including both physical interactions and functional associations. In the following we will refer to this network as the STRING network.

STRING 7 integrates known interactions coming from interaction databases, such as MINT, HPRD, BioGRID, DIP, Reactome, BIND, and KEGG, as well as text retrieved from PubMed abstracts and other scientific resources [13]. The STRING network v7.1 for the mouse organism contains 16,566 genes and 820,177 interactions with an average node degree of 49.5 . STRING 8 [14] includes beside the included databases from the previous release updated databases and new resources, such as IntAct, NCI-Nature Pathway Interaction Database and Gene Ontology protein complexes. The STRING network v8.2 for the mouse organism contains 24,442 genes and 1,405,375 interactions with an average node degree of 57.5 . It has to be noted that the two STRING versions share only 375,682 interactions representing $46 \%$ and $27 \%$ of the v7.1 and v8.2 network sizes respectively, which leads to the fact that these two releases are very different and therefore not comparable. However, we have to mention that these numbers are estimates due to mapping of gene ids.

In addition to two releases of the STRING network (versions 7.1 and 8.2), we have also obtained a proteinprotein interaction (PPI) network for mouse from BioGRID (version 2.0.61), a repository for physical and genetic interactions derived from literature and highthroughput experiments [15]. The BioGRID v2.0.61 for the mouse organism contains 1,417 genes and 2,026 interactions with an average node degree of 2.5.

We have further used a second PPI network for mouse that was derived from I2 D (Interologous Interaction Database) which is a database integrating existing curation, such as IntAct, BIND, DIP, MINT, and HPRD, high-throughput and predicted interactions [16]. The I2 D v1.72 for the mouse organism contains 10,867 genes and 79,088 interactions with an average node degree of 10.6 . 
Table 2 shows an overview about the global network properties of underlying databases.

In this study, we have applied network-based strategies to prioritize candidate genes. Further, we have compared the performance of the ranking strategies on both functional association (STRING network) and PPI networks using the BioGRID network and the I2 D network as representatives.

In Nitsch et al. 2009 we hypothesized that global network-similarity measures capture relationships between disease proteins better than algorithms based on direct interactions [9]. We used graph kernels to capture global relationships within a graph, computing global similarity of two nodes as the probability of reaching one node at some time point after a random walk starting from another node. The resulting graph led to a global similarity network where an edge between two nodes did not represent a direct interaction, but rather their similarity in this network. From this similarity network, the distance network could be easily derived.

In the Kernel Ridge Regression Ranking, we have further explored the idea of using graph kernels to detect global similarities between any genes in the network by implementing different kernels and comparing their performance. Furthermore, instead of aggregating the differential expression of neighbors weighted as a function of distance, we have smoothed a candidate gene's differential expression level using kernel ridge regression, considering its most similar neighbors and their differential expression.

\section{Candidate genes}

For each data set we have selected a set of 100 candidate genes, including the knockout gene. For getting the candidates, we have chosen the knockout genes' nearest 100 genes on the chromosome based on the BioMart data mining tool which accesses data from the Ensembl Genome Browser.

We have then prioritized this set of candidate genes using four different strategies. Results were evaluated by retrieving the position of the knockout gene in the ranking list and by calculating the corresponding AUC value.

\section{Evaluation}

Evaluation of the different strategies was accomplished by ranking the candidate genes in each data set using a number of different parameter and measure settings: MAS5/RMA/GCRMA combined with log2 ratio/significant $\log 2$ ratio/test statistic as expression measure using different kernels in the Kernel Ridge Regression Ranking strategy, using different preference vector initializations, iteration numbers and diffusion parameters in the Heat Kernel Diffusion Ranking, using different thresholds for the edge weights in the Direct Neighborhood Ranking, and different initial vectors and iteration numbers in the Arnoldi Diffusion Ranking.

The performance was assessed based on the position of the knockout gene in the ranking list. Ideally, the knockout gene should appear in the top of the ranking list based on the hypothesis that this gene is causing all the disruption in the expression of the genes in the network.

As evaluation measure, we have computed AUC values and the number of knockout genes that were successfully ranked in the top $10 \%$ among the candidate genes.

The AUC (Area Under the ROC Curve) is a standard measure of the performance of the ranking algorithm and assesses its ability to separate the two classes "positive ranked" (genes that are highly ranked) and "negative ranked" (genes that are lowly ranked). The corresponding ROC (Receiver Operating Characteristic) curve is achieved by imposing and varying a threshold (from rank 1 to $\mathrm{n}$ ) to separate these two classes, which leads to true positive rates and false positive rates for each threshold $k$. To obtain the ROC curve, the FPRs (false positive rate) are plotted against the TPRs (true positive rate). Following Fawcett (2006) the AUC is equivalent to the probability that a classifier will rank a randomly chosen positive instance higher than a randomly chosen negative instance [42]. This is equivalent to the Wilcoxon test of ranks [43]. For instance, an AUC value of $100 \%$ indicates that every knockout gene ranks in the first position, whereas an AUC value of $50 \%$ means that the knockout genes rank randomly.

\section{Ranking Strategies}

\section{Simple Expression Ranking as a Baseline}

A standard procedure in genetics to analyze candidate genes is to assess the expression level of a candidate gene in patient derived material against wild type. Candidates for which a significant difference is observed between the two groups are considered promising (see for example [20-22]). The higher a candidate's $\log 2$ ratio, the higher is its position in the ranking. This simple comparison of $\log 2$ ratios is our baseline in this study.

1. Strategy: Kernel Ridge Regression Ranking Consider a given weighted and undirected graph $G$ With symmetric weights $w_{i j} \geq 0$ between nodes $i$ and $j$. The weight $w_{i j}$ increases with the importance of the relation between nodes $i$ and $j$ : the larger its value, the easier the communication through the edge. Let $A$ be the Adjacency matrix with $a_{i j}=w_{i j}$ if the nodes $i$ and $j$ are connected and $a_{i j}=0$ otherwise. The Laplacian matrix $L$ Of $G$ is defined As $L=D-A$ With $D=\operatorname{diag}\left(a_{i}\right)=\sum_{j=1}^{n} a_{i j}$. L is symmetric and positive semidefinite. 
The Laplacian Exponential Diffusion Kernel was introduced by Kondor and Lafferty (2002) [44] as

$$
K_{L E D}=\lim _{n \rightarrow \infty}\left(I+\frac{\alpha L}{n}\right)^{n}=e^{\alpha L}
$$

whereby $\alpha$ is the diffusion parameter that determines the degree of diffusion. For a Laplacian matrix, $e^{\alpha L}$ is always positive definite and can thus be used as a kernel matrix. It can be seen as a random walk, starting from a node and transitioning to neighboring nodes with probability $\alpha$. In our application we have applied a negative diffusion parameter, i.e. $K_{L E D}=e^{-\alpha L}$.

The Regularized Laplacian Kernel was introduced by Smola E Kondor (2003) and Fouss et al. (2006) $[45,46]$ as

$$
K_{R L}=\sum_{k=0}^{\infty} \alpha^{k}(-L)^{k}=(I+\alpha L)^{-1} .
$$

The Regularized Commute time Kernel was introduced by Fouss et al. 2006 [46] as

$$
K_{R C T}=(D-\alpha A)^{-1} .
$$

Following the kernel computation, normalization and centering procedures are applied to obtain a valid Similarity Network whose values vary between 0 and 1 :

$$
\begin{aligned}
& \text { Normalization : } K_{N}=D^{*} K D^{*}, D^{*}=\frac{1}{\sqrt{D}}, D_{i i}=k\left(x_{i}, x_{i}\right) \\
& \text { Centering: } K_{C}=K_{N}-\frac{1}{n} j j^{T} K_{N}-\frac{1}{n} K_{N} j j^{T}+\frac{1}{n^{2}}\left(j^{T} K_{N} j\right) j j^{T}
\end{aligned}
$$

We have defined a neighborhood of a gene as follows. First, it must contain at least one gene other the requested gene. Second, we have limited the amount of neighbors to 50 (see results and parameter tuning in Table S1a (see additional file 1)). Furthermore, we can extract the similarities between genes from the Similarity Network. As a consequence, we obtain for each candidate gene a neighborhood consisting of the 2 to 50 most similar genes in the network that are considered to be influenced in their expression by the candidate gene.

For a given (semi) positive definite kernel matrix $K \in$ $R^{n}$ and the vector of response variables $Y \in R^{n}$, Saunders et al. (1998) [47] and Cawley et al. (2006) [48] have defined the regression problem $\hat{y}=\sum_{i=1}^{n} a_{i} K\left(x_{i}, x\right)$ with weights $a \in R^{n}$ in the Regularization. Theory as

$$
\min _{a}\|Y-K a\|_{2}^{2}+\frac{\lambda}{2} a^{T} a
$$

with $\lambda$ as a parameter defining the degree of smoothness in regression. Taking conditions for optimality $Y=$ $(K+\lambda I) a$, the solution vector $a^{*}$ is

$$
a^{*}=(K+\lambda I)^{-1} Y .
$$

For a new given $x$ it follows

$$
\hat{y}=\sum_{i=1}^{n} a_{i}^{*} K_{x}\left(x_{i}, x\right)
$$

where $K_{x}$ is a $n \times 1$ vector containing the kernel evaluations between the candidate point $x$ and $n$ neighboring points (the neighborhood of $x$ ). The resulting value of $\hat{y}$ can be seen as a smoothed differential expression value for the candidate gene $x$ having $n$ neighbors with expression values $Y$.

For each data set all candidates were ranked based on their smoothed differential expression values. A candidate gene with a strongly differentially expressed neighborhood will obtain a smoothed differential expression value that is larger than its original differential expression level derived from the microarray experiment. On the other hand, a candidate with a low differentially expressed neighborhood will obtain a small smoothed differential expression value, even if its original differential expression level had been large.

2. Strategy: Heat Kernel Diffusion Ranking The Heat Kernel Diffusion Ranking approach prioritizes the candidate genes by diffusing the differential expression values of the candidate genes through the network based on the confidence scores of the associations/interactions. We have applied the Heat Kernel rank introduced by [10] and recently used by [25] to unravel relevant regulators in the Saccharomyces cerevisiae regulatory network.

Given a graph $G$, the transition probability matrix $W$ of a random walk on $G$ is defined as $W=D^{-1} A$. Consider $L=I-W$. Given a parameter $\alpha$, establishing the diffusion rate, and a preference vector $p_{0}$, expressing the initial relevance score of each node, the ranking $p_{\alpha}$ is given by

$$
p_{\alpha}=\sum_{k=0}^{\infty} \frac{(-\alpha)^{k}}{k !} p_{0} L^{k}=p_{0} e^{-\alpha L}
$$

We have used the discrete approximation by Yang et al. (2007):

$$
p_{\alpha}=p_{0}\left(I+\frac{-\alpha}{N} L\right)^{N}
$$

with $N$ being the number of iterations [24]. This iterative diffusion can be regarded as a random walk through 
the network which is comparable to the Laplacian Exponential Diffusion Kernel (see equation (1)). However, the Heat Kernel rank considers only an initialized preference vector, whereas the Kernel Ridge Regression Ranking uses the Exponential Diffusion Kernel to solve a regression problem.

Following Francisco et al. (2009), performing reduced number of iterations is usually sufficient for ranking purposes using the Heat Kernel rank [25]. In fact, we reach a considerably good performance already after few iterations in our application (see Results section).

We have initialized the preference vector $p_{0}$ with the differential expression levels coming from a data set from our benchmark. The resulting heat diffusion rank vector $p_{\alpha}$ contains a score for every candidate gene based on the heat diffusion random walk of its expression level through the network.

3. Strategy: Arnoldi Diffusion Ranking Based on a functional association or PPI network we have computed network diffusion based on a Kyrlov Space method, namely the Arnoldi algorithm, as presented in [11]. The Arnoldi algorithm projects the exponential of a large matrix (here, the STRING network, the BioGRID network, or the I2 D network) onto a small Krylov subspace and approximates the matrix exponential operation $e^{A} v$ as

$$
e^{A} v \approx p_{m-1}(A) v
$$

where $A$ is a matrix of dimension $N, v$ is a nonzero vector and $p_{m-1}$ is a polynomial of degree $m-1$. Since this approximation is an element of the Krylov subspace $K_{m}$ $\equiv \operatorname{span}\left\{v, A v, \ldots, A^{m-1} v\right\}$, the problem was reformulated by Saad (1992) as that of finding an element of $K_{m}$ that approximates $u=e^{A} v[11]$. Based on the Krylov subspace $K_{m}$ and the Hessenberg matrix $H_{m}$ of dimension $n \times n, u$ can be computed by

$$
u=e^{A} v \approx\|v\| \cdot V_{m} e^{\alpha H_{m}} e_{1} .
$$

In this study, we want to compute the steady state vector of a random walk based on the STRING network which will be an approximation of the Laplacian Exponential Diffusion Kernel presented in equation (1) as $K=e^{\alpha L}$ with $L=I--D^{-1} A$ (see above). The kernel matrix $K$ can be seen as a random walk on the graph with transition probability $\alpha$, and its ith column vector represents the steady state probability of a random walk starting at node $i$ with a sufficient number of steps leading to convergence. Then, $K_{i j}$ is the probability of stopping at node $j$ having started at node $i$ after infinite time steps. The column vector $i$ of $K$ can be written as

$$
K e_{i}=e^{\alpha L} e_{i}
$$

where $e_{i}=[0, \ldots 0,1,0, \ldots, 0]^{\prime}$ is a zero vector with a 1 at position $i$.

To compute the approximation of the matrix exponential operation $e^{A} v$ (see equation (12)) considering expression levels for our application, we have initialized the starting vector $v$ with differential expression levels coming from a data set from our benchmark. After running the Arnoldi Algorithm the resulting vector $V_{m}$ and the corresponding Hessenberg matrix $H_{m}$ of dimension $m$ (coming from the Krylov subspace) lead to the resulting vector $u=e^{A} v$ (see equation (13)) reflecting the steady state probability of reaching node $j$ after infinite time steps by starting at the same time point from all nodes that have been initialized in starting vector $v$. The parameter $m$ is the dimension of the Kyrlov subspace and corresponds to the number of iterations in the Arnoldi approximation of network diffusion. The larger $m$, the more accurate is the approximation of network diffusion.

4. Strategy: Direct Neighborhood Ranking In the Direct Neighborhood Ranking, we have used a functional association or PPI network directly to capture a neighborhood for a candidate gene without considering any diffusion over the network. A candidate's neighborhood contains all genes $j$ directly connected to the candidate $i$ in the network with weight $w_{i j}$, representing the probability that an association exists in reality.

Let $x_{i}$ be the differential expression level of candidate gene $i, a$ the weighting parameter that determines the influence of the candidate's expression and its neighborhood's expression, $\varepsilon$ the threshold defining the minimum edge weight between the candidate gene and a neighbor, and $N$ the number of neighboring genes of candidate gene $i$ for which weight $w_{i j}$ is larger then $\varepsilon$. Then the score is computed by

$$
\hat{x}_{i}=a \cdot x_{i}+(1-a) \cdot \frac{\sum_{\left\{j \neq i, j: w_{i j}>\varepsilon\right\}^{x}} x_{j}}{N}
$$

\section{Additional material}

Additional file 1: Supplementary Tables. This document contains all supplementary tables mentioned in the article.

\section{Acknowledgements}

We kindly thank Ernesto lacucci for his helpful review of the manuscript. The research of DN, FO, BDM, and YM was supported by Research Council KUL (ProMeta, GOA Ambiorics, GOA MaNet, CoE EF/05/007 SymBioSys, START 1, several PhD/postdoc \& fellow grants), Flemish Government (FWO: 
PhD/postdoc grants, projects, G.0318.05 (subfunctionalization), G.0553.06 (VitamineD), G.0302.07 (SVM/Kernel), research communities (ICCOS, ANMMM, MLDM); G.0733.09 (3UTR); G.082409 (EGFR)), IWT (PhD Grants, Silicos; SBOBioFrame, SBO-MoKa, TBM-IOTA3), FOD (Cancer plans), Belgian Federal Science Policy Office (IUAP P6/25 (BioMaGNet, Bioinformatics and Modeling: from Genomes to Networks, 2007-2011)), EU-RTD (ERNSI: European Research Network on System Identification; FP7-HEALTH CHeartED). The work of JPG was supported by FCT PhD grant SFRH/BD/36586/2007.

\section{Author details}

'Department of Electrical Engineering (ESAT-SCD) Katholieke Universiteit Leuven, 3001 Leuven, Belgium. ${ }^{2}$ Knowledge Discovery and Bioinformatics (KDBIO) group, INESC-ID, Rua Alves Redol 9, 1000-029 Lisboa, Portugal. ${ }^{3}$ Instituto Superior Técnico, Technical Universityof Lisbon, Av. Rovisco Pais, 1049-001 Lisboa, Portugal.

\section{Authors' contributions}

DN developed the presented gene prioritization strategies, set up the experiments, analyzed the data and wrote the paper. JPG participated in writing the paper and in developing the presented gene prioritization strategies. FO participated in developing the presented gene prioritization strategies. YM and BDM supervised the project. All authors read and approved the final manuscript.

Received: 27 May 2010 Accepted: 14 September 2010 Published: 14 September 2010

\section{References}

1. Aerts S, Lambrechts D, Maity S, Van Loo P, Coessens B, et al: Gene prioritization through genomic data fusion. Nat Biotech 2006, 24(5):537-44.

2. Köhler $S$, Bauer $S$, Horn D, Robinson PN: Walking the interactome for prioritization of candidate disease genes. Am J Hum Genet 2008, 82(4):949-58.

3. Franke $L$, van Bakel $H$, Fokkens $L$, de Jong ED, Egmont-Petersen $M$, et al: Reconstruction of a functional human gene network, with an application for prioritizing positional candidate genes. Am J Hum Genet 2006, 78(6):1011-25.

4. Lage K, Karlberg EO, Storling ZM, Olason PI, Pedersen AG, et al: A human phenome-interactome network of protein complexes implicated in genetic disorders. Nat Biotech 2007, 25(3):309-16.

5. Chen J, Aronow BJ, Jegga AG: Disease candidate gene identification and prioritization using protein interaction networks. BMC Bioinformatics 2009, 27(10):73.

6. Subramanian A, Tamayo P, Mootha VK, Mukherjee S, Ebert BL, et al: Gene set enrichment analysis: a knowledge-based approach for interpreting genome-wide expression profiles. Proc Natl Acad Sci USA 2005, 102(43):15545-50.

7. Liu M, Liberzon A, Kong SW, Lai WR, Park PJ, et al: Network-Based Analysis of Type 2 Diabetes. PLoS Genet 2007, 15;3(6):e96.

8. Tranchevent LC, Capdevila FB, Nitsch D, De Moor B, De Causmaecker P, et al: A guide to web tools to prioritize candidate genes. Briefings in Bioinformatics 2010.

9. Nitsch D, Tranchevent LC, Thienpont B, Thorrez L, Van Esch H, et al: Network Analysis of Differential Expression for the Identification of Disease-Causing Genes. PLOS ONE 2009, 4(5):e5526.

10. Chung F, Yau ST: Coverings, heat kernels and spanning trees. Electronic Journal of Combinatorics 1999, 6.

11. Saad $Y$ : Analysis of some Krylov subspace approximations to the matrix exponential operator. SIAM Journal on Numerical Analysis (SINUM) 1992, 29(1):209-228.

12. Barrett T, Troup DB, Wilhite SE, Ledoux P, Rudnev D, et al: NCBI GEO: mining tens of millions of expression profiles - database and tools update. Nucleic Acids Res 2007, , 35 Database: D760-5.

13. von Mering C, Jensen LJ, Kuhn M, Chaffron S, Doerks T, et al: STRING 7 recent developments in the integration and prediction of protein interactions. Nucleic Acids Res 2007, 35:D358-62.

14. Jensen $L$, Kuhn M, Stark M, Chaffron S, Creevey C, et al: STRING 8 - a global view on proteins and their functional interactions in 630 organisms. Nucleic Acids Res 2009, , 37 Database: D412-6.
15. Stark C, Breitkreutz BJ, Reguly T, Boucher L, Breitkreutz A, et al: Biogrid: A General Repository for Interaction Datasets. Nucleic Acids Res 2006, 34: D535-9.

16. Brown KR, Jurisica I: Online predicted human interaction database. Bioinformatics 2005, 21(9):2076-82.

17. Chuang HY, Lee E, Liu YT, Lee D, Ideker T: Network-based classification of breast cancer metastasis. Mol Syst Biol 2007, 3:140.

18. Gupta R, Agrawal S, Rao N, Tian Z, Kuang R, et al: Integrative Biomarker Discovery for Breast Cancer Metastasis from Gene Expression and Protein Interaction Data Using Error-tolerant Pattern Mining. Proceedings of the International Conference on Bioinformatics and Computational Biology (BICOB) 2010.

19. Nibbe RK, Koyutürk M, Chance MR: An Integrative -omics Approach to Identify Functional Sub-Networks in Human Colorectal Cancer. PLoS Comput Biol 2010, 6(1):e1000639.

20. Park JW, Cai J, McIntosh I, Jabs EW, Fallin MD, et al: High throughput SNP and expression analyses of candidate genes for non-syndromic oral clefts. J Med Genet 2006, 43(7):598-608.

21. Mirkin S, Arslan M, Churikov D, Corica A, Diaz Jl, et al: In search of candidate genes critically expressed in the human endometrium during the window of implantation. Hum Reprod 2005, 20:2104-2117.

22. Zieker D, Fehrenbach E, Dietzsch J, Fliegner J, Waidmann M, et al: cDNA microarray analysis reveals novel candidate genes expressed in human peripheral blood following exhaustive exercise. Physiol Genomics 2005, 23(3):287-94.

23. Baldi P, Long AD: A Bayesian Framework for the Analysis of Microarray Expression Data: Regularized t-Test and Statistical Inferences of Gene Changes. Bioinformatics 2001, 17(6):509-519.

24. Yang H, King I, Lyu MR: Diffusionrank: a possible penicillin for web spamming. In Proceedings of the 30th annual international ACM SIGIR conference on Research and development in information retrieval: 23-27 July 2007, Amsterdam. Edited by: Kraaij W, de Vries AP, Clarke CLA, Fuhr N, Kando N. ACM; 2007:431.

25. Francisco AP, Goncalves JP, Madeira SC, Oliveira AL: Using personalized ranking to unravel relevant regulations in the Saccharomyces cerevisiae regulatory network. Proceedings of Jornadas de Bioinformatica 2009, 3-6 November 2009, Lisbon.

26. Choe SE, Boutros M, Michelson AM, Church GM, Halfon MS: Preferred analysis methods for Affymetrix GeneChips revealed by a wholly defined control dataset. Genome Biol 2005, 6(2):R16.

27. Harr B, Schlötterer C: Comparison of algorithm $s$ for the analysis of Affymetrix microarray data as evaluated by co-expression of genes in known operons. Nucleic Acids Res 2006, 34(2):e8.

28. Lim WK, Wang K, Lefebvre C, Califano A: Comparative analysis of microarray normalization procedures: effects on reverse engineering gene networks. Bioinformatics 2007, 23(13):i282-8.

29. Vardhanabhuti S, Blakemore SJ, Clark SM, Ghosh S, Stephens RJ, et al: A comparison of statistical tests for detecting differential expression using Affymetrix oligonucleotide microarrays. OMICS 2006, 10(4):555-66.

30. Panchenko A, Przytycka T, (eds): Protein-protein Interactions and Networks Springer-Verlag, London 2008.

31. Salwinski L, Miller CS, Smith AJ, Pettit FK, Bowie JU, et al: The Database of Interacting Proteins: 2004 update. Nucleic Acids Res 2004, , 32 Database: D449-51.

32. Bader GD, Donaldson I, Wolting C, Ouellette BF, Pawson T, et al: BIND-The Biomolecular Interaction Network Database. Nucleic Acids Res 2001, 29(1):242-5.

33. Aranda B, Achuthan $P$, Alam-Faruque $Y$, Armean I, Bridge A, et al: The IntAct molecular interaction database in 2010. Nucleic Acids Res 2009.

34. Zanzoni A, Montecchi-Palazzi L, Quondam M, Ausiello G, HelmerCitterich M, et al: MINT: a Molecular INTeraction database. FEBS Lett 2002, 513(1):135-40.

35. Razick S, Magklaras G, Donaldson IM: iRefIndex: A consolidated protein interaction database with provenance. BMC Bioinformatics 2008, 9(1):405.

36. Pagel $P$, Kovac $S$, Oesterheld M, Brauner B, Dunger-Kaltenbach I, et al: The MIPS mammalian protein-protein interaction database. . Bioinformatics 2005, 21(6):832-834.

37. Keshava Prasad TS, Goel R, Kandasamy K, Keerthikumar S, Kumar S, et al: Human Protein Reference Database - 2009 Update. Nucleic Acids Res 37: D767-72. 
38. Affymetrix: technical support documentation. [http://www.affymetrix.com/ support/technical/byproduct.affx?product=mas].

39. Irizarry RA, Hobbs B, Collin F, Beazer-Barclay YD, Antonellis K, et al: Exploration, normalization, and summaries of high density oligonucleotide array probe level data. Biostatistics 2003, 4(2):249-64

40. Wu Z, Irizarry RA: Stochastic models inspired by hybridization theory for short oligonucleotide arrays. J Comput Biol 2005, 12(6):882-93.

41. Gautier L, Cope L, Bolstad BM, Irizarry RA: affy - analysis of Affymetrix GeneChip data at the probe level. Bioinformatics 2004, 20(3):307-15.

42. Fawcett T: An introduction to ROC analysis. Pattern Recognition Letters 2006, 27:861-874.

43. Hanley JA, MCNeil BJ: The Meaning and use of the Area under a Receiver Operation Characteristic (ROC) Curve. Radiology 1982, 143:29-36.

44. Kondor Rl, Lafferty JD: Diffusion kernels on graphs and other discrete structures. In Proceedings of the Nineteenth International Conference on Machine Learning (ICML): 8-12 July 2002, Sydney. Edited by: Sammut C, Hoffmann AG. Morgan Kaufmann; 2002:315-322.

45. Smola AJ, Kondor R: Kernels and regularization on graphs. In Proceedings of the 16th Annual Conference on Learning Theory (COLT): 24-27 August 2003, Washington DC. Edited by: Schölkopf B, Warmuth M. Springer Verlag; 2003:.

46. Fouss F, Yen L, Pirotte A, Saerens M: An Experimental Investigation of Graph Kernels on a Collaborative Recommendation Task. Proceedings of the 6th IEEE International Conference on Data Mining (ICDM): 18-22 December 2006, Hong Kong IEEE Computer Society 2006, 863-868.

47. Saunders C, Gammerman A, Vovk V: Ridge Regression Learning Algorithm in Dual Variables. In Proceedings of the 15th International Conference on Machine Learning (ICML): 24-27 July 1998, Madison. Edited by: Shavlik JW. Morgan Kaufmann Publishers; 1998:

48. Cawley GC, Talbot NLC, Chapelle O: Estimating Predictive Variances with Kernel Ridge Regression. In Proceedings of Machine Learning Challenges: First PASCAL Machine Learning Challenges Workshop (MLCW): 11-13 July 2005, Southampton. Edited by: Quinonero-Candela J, Dagan I, Magnini B, D'AlchéBuc F. Springer Verlag; 2006:56-77.

doi:10.1186/1471-2105-11-460

Cite this article as: Nitsch et al:: Candidate gene prioritization by network analysis of differential expression using machine learning approaches. BMC Bioinformatics 2010 11:460.

\section{Submit your next manuscript to BioMed Central and take full advantage of:}

- Convenient online submission

- Thorough peer review

- No space constraints or color figure charges

- Immediate publication on acceptance

- Inclusion in PubMed, CAS, Scopus and Google Scholar

- Research which is freely available for redistribution

Submit your manuscript at www.biomedcentral.com/submit
Biomed Central 